\title{
Determination of Independent Contact Regions on Discretized 3D Objects*
}

\author{
Máximo A. Roa and Raúl Suárez \\ Institute of Industrial and Control Engineering (IOC) \\ Technical University of Catalonia (UPC), Barcelona, Spain \\ Emails:maximo.roa@upc.edu,raul.suarez@upc.edu
}

\begin{abstract}
This paper deals with the problem of determining independent contact regions on a 3D object boundary such that a seven finger frictionless grasp with a contact point in each region assures a force-closure grasp on the object, independently of the exact position of the contact points. These regions provide robustness in front of finger positioning errors in grasp and fixture applications. The object's surface is discretized in a cloud of points, so the procedure is applicable to objects of any arbitrary shape. The procedure finds an initial force-closure grasp that is iteratively improved through an oriented search procedure; once a locally optimum grasp has been reached, the independent contact regions are computed. The procedure has been implemented and application examples are included in the paper.
\end{abstract}

Index Terms-Grasp planning, force-closure grasps, independent contact regions.

\section{INTRODUCTION}

The determination of contact locations to immobilize the object despite external disturbances has been a topic of great interest in grasping, manipulation and fixturing. The contact locations are characterized by the properties of form or force-closure [1]. In form-closure the position of the contacts ensures the object immobility; this property is mostly used when the task requires a robust grasp not relying on friction, e.g. the fixture of objects to be manufactured or inspected. On the other hand, force-closure is achieved when the forces applied at the contact points ensure the object immobility; it is specially used in grasping and manipulation of objects with a low number of frictional contacts using mechanical grippers or hands.

Several algorithms have been developed to determine precision grasps (i.e. grasps formed by a set of finger contact points on the object's surface), with different number of fingers and satisfying the form or force-closure condition in 2D polygonal [2], non-polygonal [3] or discrete objects [4], 3D polyhedral objects [5] [6], objects with smooth curved surfaces [7] or 3D discretized objects [8]. Precision grasps require a good precision in the finger placements; however, in a real execution the actual and the theoretical grasp may differ due to fingers positioning errors. Nguyen [9] introduced the concept of independent contact regions (ICRs)

\footnotetext{
* This work was partially supported by the CICYT projects DPI2004-03104 and DPI2005-00112, and Acción Integrada HI2005-0290.

Máximo Roa works at the National University of Colombia. He is supported by $\mathrm{Al} \beta \mathrm{an}$, the European Union Programme of High Level Scholarships for Latin America, scholarship No.E04D039103CO.
}

in order to provide robustness to the grasp in front of positioning errors. ICRs are regions on the object boundary such that the fingers can be positioned on them assuring a force-closure (FC) grasp, with independence of the exact position of each finger.

The determination of ICRs was initially addressed for two frictional contacts on polygonal and polyhedral objects, and with four frictionless contacts on 2D polygonal objects [9]. The concept was extended to three-finger grasps of polygonal objects [10] and to four-finger grasps on polyhedral objects [5]. The notion of ICR has also been used to determine contact regions on 3D objects based on initial examples, although the results depend on the choice of the example [11]. Recently, the computation of ICRs for 2D discrete objects has also been addressed [12]; however, the determination of ICRs on 3D discrete objects has not been directly tackled yet.

This paper deals with the problem of determining independent contact regions on a 3D object boundary for a seven finger frictionless grasp, such that the ICRs assure a FC grasp with a controlled minimum quality. The proposed approach has three phases. The first phase finds an initial force-closure grasp with an algorithm similar to the one proposed in [8], but using a different FC test that decreases the search complexity. The second phase improves the initial grasp through an oriented search procedure. The optimization is carried out using a quality measure equivalent to the largest perturbation wrench that the grasp can resist, with independence of the perturbation direction [13]; it is one of the most popular grasp quality measures. The optimization is carried out to obtain a locally optimum FC grasp. Finally, the third phase computes the ICRs from the locally optimum grasp obtained in the previous phase. The proposed approach does not take into account the kinematical constraints imposed by the mechanical hand or gripper.

A work in this line [11] presents a procedure to compute a family of grasps for 3D objects that keep a fraction of the quality of the grasp in an initial example; the quality measure is the reciprocal of the sum of magnitudes of the normal contact forces required to achieve the worst case wrench in a task set [14]. However, the selection of a good initial example remains as a critical step; this initial grasp is provided here with a procedure assuring a locally optimum grasp.

This paper is organized as follows. Section II presents the 
approach to compute locally optimum frictionless FC grasps (phases 1 ant 2), and Section III presents the procedure to compute the independent contact regions (phase 3). The algorithms have been implemented, and Section IV shows the results of their application to different objects. Finally, Section V presents the conclusions of the work.

\section{LOCALLY OPTIMUM FORCE-CLOSURE GRASP}

\section{A. Object and contact models}

To compute the independent contact regions for a frictionless grasp on an arbitrary 3D object, the following assumptions are considered:

- The external surface of the object is represented with a mesh $\Omega$ of points, described by position vectors $\boldsymbol{p}_{i}$ measured with respect to a reference system located in the center of mass $(C M)$ of the object. Each point has an associated unitary normal direction $\hat{\boldsymbol{n}}_{i}$ pointing towards the interior of the object.

- The number of points in $\Omega$ is large enough to accurately represent the surface of the object.

- Each point on the surface of the object is connected with three neighboring points.

Seven frictionless contacts are necessary and may be sufficient to hold a 3D object with a FC grasp, provided that the object has no rotational symmetries [15]. With frictionless contact points, the grasp forces can only be applied in the direction normal to the object surface. A force $\boldsymbol{f}_{i}=\alpha_{i} \hat{\boldsymbol{n}}_{i}$ applied on the object at the point $\boldsymbol{p}_{i}$ generates a torque $\boldsymbol{\tau}_{i}=\boldsymbol{p}_{i} \times \boldsymbol{f}_{i}$ with respect to $C M$, with $\alpha_{i}$ being a nonnegative value representing the magnitude of the grasping force. The force and the torque are grouped together in a wrench vector (also known as generalized force vector) given by

$$
\tilde{\boldsymbol{\omega}}_{i}=\left(\begin{array}{c}
\boldsymbol{f}_{i} \\
\boldsymbol{\tau}_{i}
\end{array}\right)=\alpha_{i}\left(\begin{array}{c}
\hat{\boldsymbol{n}}_{i} \\
\boldsymbol{p}_{i} \times \hat{\boldsymbol{n}}_{i}
\end{array}\right)
$$

For a given grasp $G=\left\{\boldsymbol{p}_{1}, \boldsymbol{p}_{2}, \ldots, \boldsymbol{p}_{7}\right\}$, the wrenches applied at the contact points on the object are grouped in a wrench set $W=\left\{\boldsymbol{\omega}_{1}, \boldsymbol{\omega}_{2}, \ldots, \boldsymbol{\omega}_{7}\right\}$, where each $\boldsymbol{\omega}_{i}$, $i=1, \ldots, 7$, is called a primitive contact wrench when $\alpha_{i}=1$. Since each physical point $\boldsymbol{p}_{i}$ in the set $\Omega$ has a corresponding primitive wrench $\omega_{i}$ in the wrench space, both of them will be used to indicate a grasp point.

\section{B. Force-closure test}

Several criteria have been proposed to test the forceclosure property in a particular grasp. A necessary and sufficient condition for the existence of a FC grasp is that the origin of the wrench space lies strictly inside the convex hull $(\mathrm{CH})$ of the primitive contact wrenches [16]. Querying whether the origin lies inside the $C H$ is also equivalent to a ray-shooting problem, solved as a linear programming problem [17]. The FC test used in this work is based on the following lemma.

Lemma 1: Let $G$ be a grasp with a set $W$ of primitive contact wrenches, $\mathcal{I}$ the set of strictly interior points of

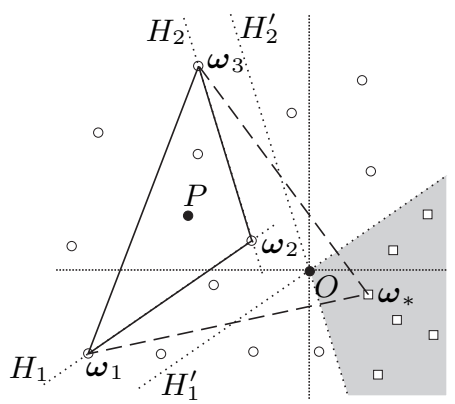

Fig. 1. The grasp with wrench set $W=\left\{\boldsymbol{\omega}_{1}, \boldsymbol{\omega}_{2}, \boldsymbol{\omega}_{3}\right\}$ (with $C H$ represented in continuous lines) is non-FC. The subset of points to be replaced is $G_{R}^{k}=\left\{\boldsymbol{\omega}_{2}\right\}$. Wrenches in the gray zone (depicted as white squares) form the set $\Omega_{C}^{k}$ of wrenches that may provide a FC grasp. The grasp with wrench set $W^{*}=\left\{\boldsymbol{\omega}_{1}, \boldsymbol{\omega}_{*}, \boldsymbol{\omega}_{3}\right\}$ (with $C H$ represented in discontinuous lines) using a candidate point $\boldsymbol{\omega}_{*}$ is a FC grasp.

$C H(W)$, and $H$ a boundary hyperplane of $C H(W)$ (i.e. a hyperplane containing one of the facets of $C H(W)$ ). The origin $O$ of the wrench space satisfies $O \in \mathcal{I}$ iff any $P \in \mathcal{I}$ and $O$ lie in the same half-space for every $H$ of $C H(W)$.

From Lemma 1 , checking whether a given point $P \in \mathcal{I}$ and the origin $O$ lie in the same half-space defined by each boundary hyperplane $H$ of $C H(W)$ is enough to prove whether $O$ lies inside $C H(W)$, i.e. to prove whether the grasp $G$ is FC. $P$ is chosen as the centroid of the primitive contact wrenches, which is always an interior point of $C H(W)$. Then, the FC test checks whether the centroid $P$ and the origin $O$ lie on the same side for all the boundary hyperplanes of $\mathrm{CH}(W)$; Fig. 1 illustrates the concept with a FC grasp and a non FC grasp in a hypothetical 2D wrench space (the actual wrench space is 6-dimensional).

\section{First phase: getting one force-closure grasp}

The main ideas of the algorithm used in the first phase are similar to those used in [8]. The algorithm generates an initial grasp $G^{1}$ selecting seven random points from $\Omega$; builds the corresponding wrench set $W^{1}$ and checks whether the points form a FC grasp. If they do, then the algorithm finishes. If $G^{1}$ is not a FC grasp, then an oriented search is performed, based on separating hyperplanes that define a subset $\Omega_{C}^{1}$ containing candidate points to replace one of the current points in $G^{1}$. The steps in the algorithm are:

Algorithm 1: Search of a FC grasp

1) Generate a random initial grasp $G^{k}=\left\{\boldsymbol{\omega}_{1}, \ldots, \boldsymbol{\omega}_{7}\right\}$, $k=1$.

2) Form the corresponding wrench set $W^{k}$.

3) Check whether $G^{k}$ is a FC grasp; if so, the algorithm finishes and returns $G^{k}$. If $G^{k}$ is not a FC grasp, the search procedure iteratively tries to improve the grasp by changing one of the points in $G^{k}$, looking for a reduction in the distance between $C H(W)$ and the origin $O$, as follows in steps 4 to 6 .

4) Find the subset $G_{R}^{k}$ of grasp points in $G^{k}$ that may be replaced. This subset contains all the wrenches in $W$ that simultaneously belong to all the hyperplanes that produce 


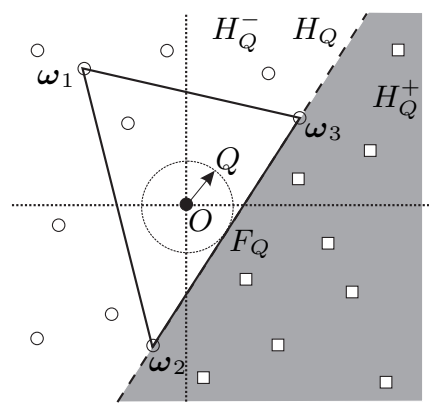

Fig. 2. Selection of the subset $\Omega_{C}^{k}$ of candidate points (depicted as white squares in the gray area) that may improve the grasp quality; in this example, $F_{Q}=\overline{\boldsymbol{\omega}_{2} \boldsymbol{\omega}_{3}}$.

the FC test failure (hereafter called critical hyperplanes). For instance, in Fig. 1 two hyperplanes, $H_{1}$ and $H_{2}$, produce the FC test failure, and $G_{R}^{k}=\left\{\boldsymbol{\omega}_{2}\right\}$.

5) Build the subset $\Omega_{C}^{k}$ with candidate points to replace one of the points in $G_{R}^{k}$. This subset is determined using hyperplanes passing through the origin and parallel to the critical hyperplanes; the candidate points are those than simultaneously lie in the opposite side of $P$ with respect to those hyperplanes. In Fig. 1, wrenches that lie in the gray zone, determined by hyperplanes $H_{1}^{\prime}$ and $H_{2}^{\prime}$, belong to $\Omega_{C}^{k}$.

6) Replace one point in $G_{R}^{k}$ with a point from $\Omega_{C}^{k}$. A point $\boldsymbol{\omega}_{*}$ is randomly picked from $\Omega_{C}^{k}$; then, $\boldsymbol{\omega}_{*}$ replaces the closest point in $G_{R}^{k}$. The candidate grasp $G^{*}$ is formed with that replacement (in the example in Fig. 1, $\left.G^{*}=\left\{\boldsymbol{\omega}_{1}, \boldsymbol{\omega}_{*}, \boldsymbol{\omega}_{3}\right\}\right)$, and the centroid $P^{*}$ and the distance $\overline{P^{*} O}$ are computed for the candidate grasp. If for any candidate $G^{*}$ the relation $\overline{P^{*} O}<\overline{P^{k} O}$ is satisfied, then the best-first option is taken, and the corresponding point $\boldsymbol{\omega}_{*}$ is selected as the replacement point. If all the points in $G_{R}^{k}$ have been checked out and none of them decreases the distance $\overline{P^{k} O}$, the replacement is done choosing the candidate $G^{*}$ that gives the smaller distance $\overline{P^{*} O}$. Finally, the counter $k$ is updated, the selected point is included in the new grasp $G^{k}$, and the procedure returns to Step 2.

To avoid falling in a local minimum, the generated grasps $G^{k}$ are stored, and if Step 6 gives an already considered grasp, it is discarded and the next best non-visited candidate is taken for the replacement. This consideration allows the grasp search procedure to overcome local minima until a FC grasp is found. In this sense, the algorithm is complete in the discrete domain (as the algorithm in [8] it finds a FC grasp if there is one).

\section{Second phase: finding a locally optimum grasp}

The optimization algorithm begins with an initial FC grasp obtained through the procedure described above, and the optimization is done looking for the largest perturbation wrench that the grasp can resist with independence of its direction [13]. Geometrically, that quality is the radius of the largest ball centered at the origin of the wrench space and

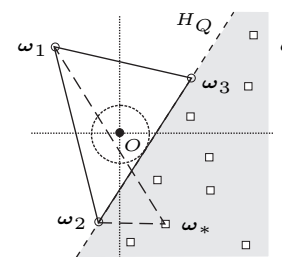

a)

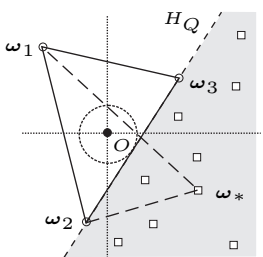

b)

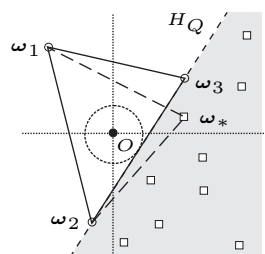

c)
Fig. 3. Possible cases for a candidate grasp in the optimization procedure: a) Non-feasible candidate grasp, b) Discarded candidate grasp, c) Feasible candidate grasp.

fully contained in $C H(W)$, i.e. it is the distance from the origin of the wrench space to the closest facet of $C H(W)$. The steps in the algorithm are:

Algorithm 2: Search of a locally optimum grasp

1) Find an initial FC grasp, $G^{k}=\left\{\boldsymbol{\omega}_{1}, \ldots, \boldsymbol{\omega}_{7}\right\}, k=1$, using Algorithm 1 presented in Subsection II-C.

2) Determine $F_{Q}$, the facet of the convex hull $C H\left(W^{k}\right)$ closest to the origin. The distance from the origin $O$ to $F_{Q}$ is the current grasp quality $Q^{k}$.

3) Build the subset $\Omega_{C}^{k}$ with the candidate points that may produce an improvement in the grasp if they replace one point in $F_{Q}$. Let $H_{Q}$ be the hyperplane containing the facet $F_{Q}$, and $H_{Q}^{+}$the open half-space defined by $H_{Q}$ that does not contain the origin $O$. The subset $\Omega_{C}^{k}$ contains the points lying in $H_{Q}^{+}$, as illustrated in Fig. 2.

4) Generate 6 candidate grasps $G_{i}^{*}, i=1, \ldots, 6$ by picking a point $\omega_{*}$ from $\Omega_{C}^{k}$ and replacing each one of the vertices defining the facet $F_{Q}$. Due to the selection procedure, all the wrenches $\omega^{*} \in \Omega_{C}^{k}$ are external points to $C H(W)$, therefore, when replacing one vertex $\boldsymbol{\omega}_{i}$ from the actual $C H$ with the candidate wrench $\boldsymbol{\omega}_{*}$, the latter will be a vertex of the new $C H$. The explicit computation of the new $\mathrm{CH}$ is not required, as its facets are constructed from the old ones replacing $\boldsymbol{\omega}_{i}$ with $\boldsymbol{\omega}_{*}$. The candidate grasps are checked for the FC property using Lemma 1. For the FC candidate grasps, the expected grasp quality $Q^{*}$ is computed; if for any candidate grasp $Q^{*}>Q^{k}$, then the candidate becomes the new grasp $G^{k}$. Fig. 3 illustrates three possible cases related with the candidate grasps; case (a) is a non-feasible grasp because it loses the FC property, case (b) is discarded because the grasp has a smaller quality than the previous one, and case (c) is a good grasp that actually improves the grasp quality, thus it becomes the grasp for the next iteration cycle. After this step, if the quality is improved then go back to Step 2. If there is no improvement in $Q^{k}$ once all the points in $\Omega_{C}^{k}$ have been considered, then a local minimum has already been reached, the algorithm finishes and returns the current grasp $G$.

\section{INDEPENDENT CONTACT REGIONS}

The computation of the independent contact regions (ICRs) ensuring a minimum grasp quality $Q$ begins with a locally optimum FC grasp. Considering the ICRs for each 


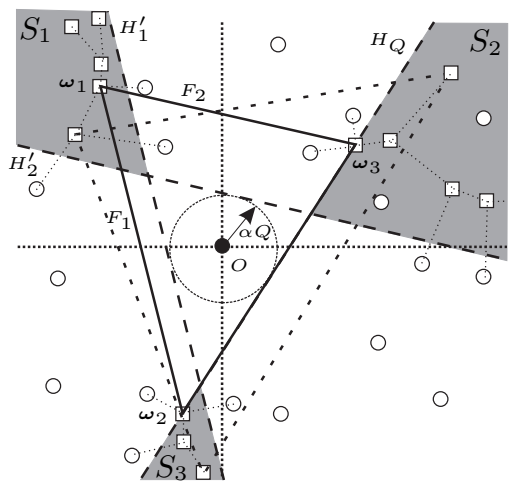

Fig. 4. Search of the independent contact regions. The hyperplanes $H_{Q}$, $H_{1}^{\prime}$ and $H_{2}^{\prime}$ define the search zones $S_{1}, S_{2}$ and $S_{3}$ (depicted in gray). The ICRs are the sets of neighboring wrenches falling in the search zone. Wrenches in each ICR are depicted as white squares, and an instance of a grasp with quality higher than $Q(\alpha=1)$ is also shown.

finger, several grasps may be formed when placing a finger in different positions inside each ICR; any of these grasps must satisfy $O \in C H(W)$. The proposed approach is based on this geometrical condition, as illustrated in Fig. 4 for a hypothetical two-dimensional wrench space. For a given FC grasp, the grasp quality $Q$ is fixed by $F_{Q}$, the facet of the convex hull closest to the origin. Six hyperplanes $H^{\prime}$ (two in the hypothetical two-dimensional wrench space), parallel to the remaining facets of the convex hull and tangent to the ball of radius $r=Q$ are then considered. These hyperplanes define $S_{i}$, the search zone containing the ICR for each wrench $\omega_{i} ; S_{i}$ is the intersection of the half-spaces defined by the hyperplanes $H^{\prime}$ that contain the wrench $\boldsymbol{\omega}_{i}$. The ICR is the set of neighboring points of $\boldsymbol{\omega}_{i}$ falling into the corresponding search zone $S_{i}$.

The procedure can also be applied to generate ICRs with contact points that produce a lower grasp quality $Q_{r}=\alpha Q$, with $0<\alpha<1$ and $Q$ the quality of the initial grasp. This is achieved considering a ball of radius $Q_{r}$ instead of $Q$ in the procedure described above. When $\alpha \rightarrow 0$, the ICRs contain FC grasps without a lower limit on the grasp quality. In fact $\alpha=0$ is a forbidden value, as it does not assure that any $C H(W)$ will strictly contain the origin $O$. The algorithm used to determine the ICRs is:

\section{Algorithm 3: Search for the independent contact regions}

1) Find a locally optimum FC grasp, $G_{o}=\left\{\boldsymbol{\omega}_{1}, \ldots, \boldsymbol{\omega}_{7}\right\}$, with the corresponding wrench set $W_{o}$, using Algorithm 2 presented in Subsection II-D.

2) Fix the minimum acceptable quality $Q_{r}=\alpha Q$.

3) Build the hyperplanes $H^{\prime}$, tangent to the ball of radius $Q_{r}$ centered at the origin, that define the search space $S_{i}, i=1, \ldots, 7$, for each grasp point.

4) Initialize $I_{i}$, the set of contiguous points forming the ICR for the grasp point $i$, as $I_{i}=\left\{\boldsymbol{\omega}_{i}\right\}$ (i.e. each ICR contains the original wrench of the set $W_{o}$ ). Label the points in each $I_{i}$ as open.

5) For each open point $\boldsymbol{\omega}_{k}$ in the set $I_{i}$, check whether the neighbor points $\boldsymbol{\omega}_{k n}$ lie into the corresponding search

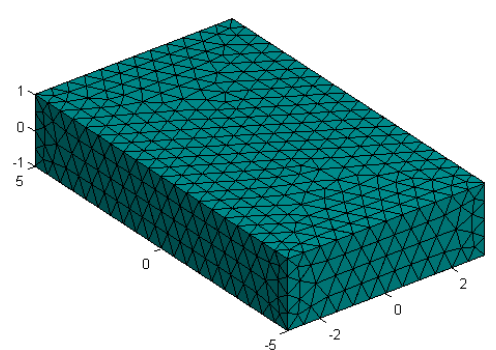

a)

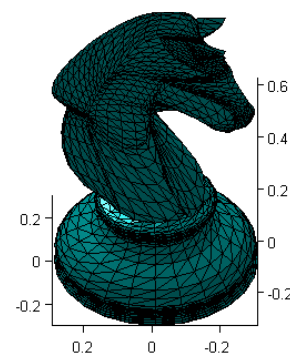

b)
Fig. 5. Objects used in the examples: a) Parallelepiped discretized with a mesh of 3422 triangles, b) Knight discretized with 4750 triangles.

space $S_{i}$. If $\boldsymbol{\omega}_{k n} \in S_{i}$ then add $\boldsymbol{\omega}_{k n}$ to $I_{i}$ and label it as open; otherwise, discard the point. Label $\boldsymbol{\omega}_{k}$ as closed.

6) If there are open points in $I_{i}$, go back to Step 5. Otherwise, the algorithm finishes, and returns the sets of points $I_{i}, i=1, \ldots, 7$, i.e. the ICRs for each finger.

Due to the geometrical construction of the procedure, the obtained ICRs depend on the used initial grasp. The size of the ICRs could be optimized changing the locally optimum grasp used to build them. This problem is not addressed in this paper, but it is an interesting issue to explore in the future.

\section{EXAMPLES}

The proposed approach to compute independent contact regions has been implemented using Matlab on a Pentium IV $3.2 \mathrm{GHz}$ computer. The performance of the algorithm is illustrated using the two objects shown in Fig. 5: a parallelepiped and a chess knight. The object surfaces are represented with triangular meshes (two triangles of the mesh are considered neighbors if they share an edge). The considered contact points $\boldsymbol{p}_{i}$ on the object surface are the centroids of the triangles in the mesh, and the corresponding surface normal directions are the directions normal to the triangles.

In the first example, the parallelepiped is described with a mesh of 3422 triangles. Fig. 6 shows an instance of the results obtained with the proposed approach. The first FC grasp, obtained with the Algorithm 1, is shown in Fig. 6a; the time elapsed to obtain this grasp was 5.1 seconds in 17 iterations. The locally optimum FC grasp, shown in Fig. 6b, was obtained with the Algorithm 2 in 24.8 seconds and 32 iterations. Fig. 6c shows the corresponding independent contact regions, obtained with Algorithm 3 in 0.25 seconds and using as minimum quality $Q_{r}=0.2168(\alpha=0.75)$. Fig. 8a plots the distance $\overline{P O}$ against the iteration number in the first phase. Fig. $8 \mathrm{~b}$ plots the grasp quality in the optimization phase, which always increases monotonically up to the locally optimum grasp. The obtained locally optimum grasp depends on the initial grasp. In the example, the initial grasp quality is 0.0102 , and the locally optimum grasp quality is 0.2891 ; the improvement factor, i.e. the ratio between the quality of the optimized grasp and the quality of the initial FC grasp is 28.4. The points within the ICRs may be combined to provide 75000 different grasps; Fig. 9 


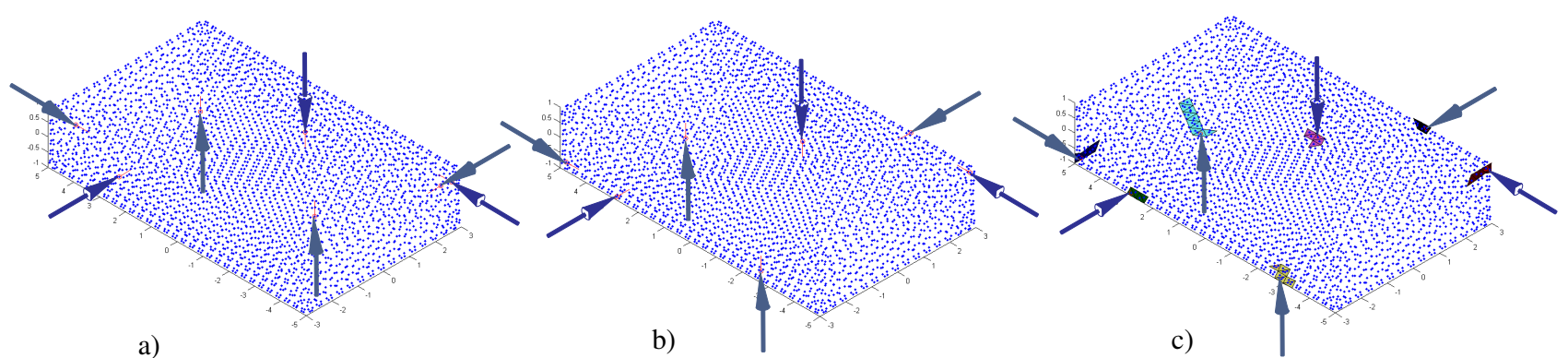

Fig. 6. Example on a parallelepiped: a) Initial FC grasp, $Q=0.0102$ (Algorithm 1), b) Locally optimum FC grasp, $Q=0.2891$ (Algorithm 2), c) Independent contact regions for each finger, $Q_{r}=0.2168$ (Algorithm 3).
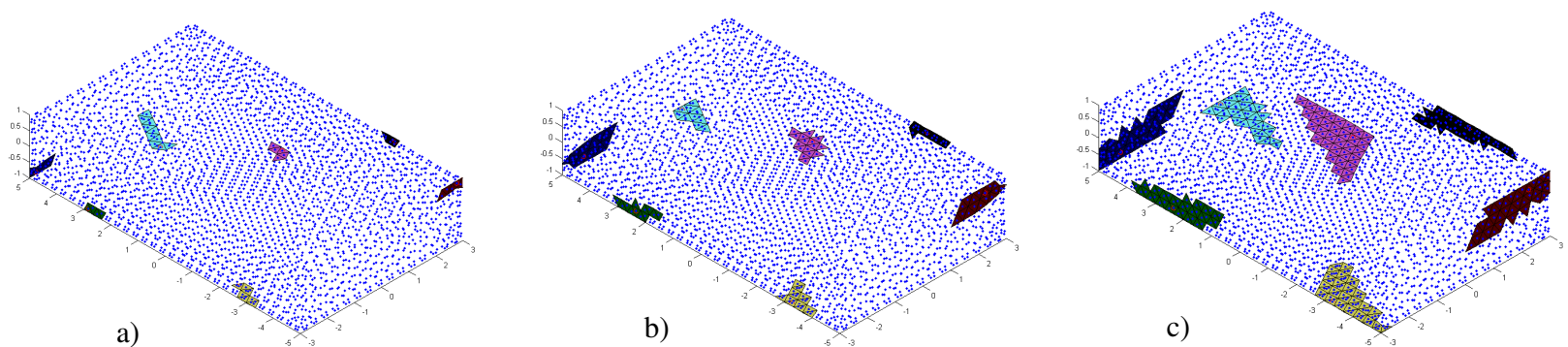

Fig. 7. Independent contact regions on the parallelepiped with different minimum quality: a) $Q_{r}=0.2168(\alpha=0.75)$, b) $Q_{r}=0.1446(\alpha=0.5)$, c) $Q_{r} \approx 0\left(\alpha=10^{-5}\right)$.

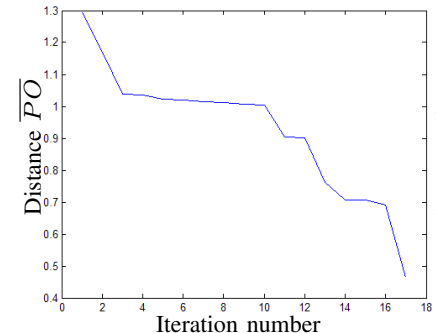

a)

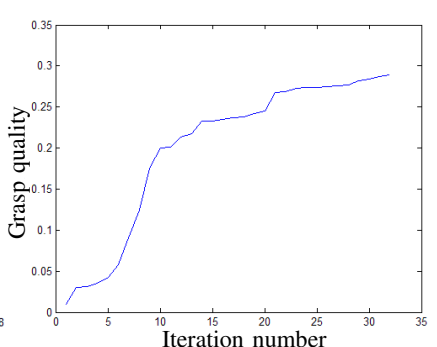

b)

Fig. 8. Performance in the search of a locally optimum FC grasp for the parallelepiped: a) Variation in the distance $\overline{P O}$, b) Increase in the grasp quality.

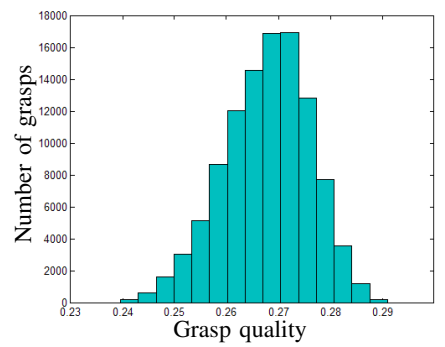

Fig. 9. Histogram with the grasp quality distribution for all the possible grasps within the independent contact region on the parallelepiped for $Q_{r}=0.2168(\alpha=0.75)$.

shows the quality distribution for all these possible grasps. Obviously, for lower minimum grasp qualities, the size of each ICR grows; Fig. 7 shows the ICRs for three different minimum grasp qualities given by $\alpha=0.75, \alpha=0.5$ and $\alpha=10^{-5} \approx 0$. In the last case, the ICRs contain points such that a finger in each region assures a FC grasp, but without a limit in the lower grasp quality.

The knight used in the second example is discretized

with 4750 triangles (Fig. 5b). Fig. 10 shows the results for an ICR search on the knight; the first FC grasp was found after 9 iterations in 5.4 seconds, the locally optimum grasp was obtained after 48 iterations in 47 seconds and the ICRs (with $Q_{r}=0.058, \alpha=0.75$ ) were computed in 0.17 seconds. The grasp qualities are 0.0003 and 0.077 for the initial and locally optimum FC grasps, respectively, with an improvement factor of 225.7. Fig. 11 illustrates the performance of Algorithms 1 and 2 in the search process. The points within the ICRs may be combined to provide 30 different grasps; Fig. 9 shows the quality distribution for all these possible grasps. Fig. 13 shows the ICRs for three different quality ratios: $\alpha=0.75, \alpha=0.5$ and $\alpha=10^{-5}$.

\section{CONCLUSIONS}

This paper proposes an integrated approach to obtain independent contact regions on 3D discretized objects with seven frictionless contacts that assure a FC grasp with a controlled minimum quality. The procedure has three main parts: the first one looks for an initial FC grasp (its main ideas were presented in [8], although a different FC test is used here). The second part optimizes the initial FC grasp with an oriented search procedure, using as a quality measure the largest perturbation wrench that the grasp can resist, with independence of the perturbation direction. The third part computes the independent contact regions around the contact locations of the locally optimum FC grasp. The algorithms were implemented and the execution results, as the examples shown in the paper, illustrate the relevance and efficiency of the approach. Although the algorithm is described just for seven frictionless fingers, it can be easily extended to determine ICRs for more fingers. The 


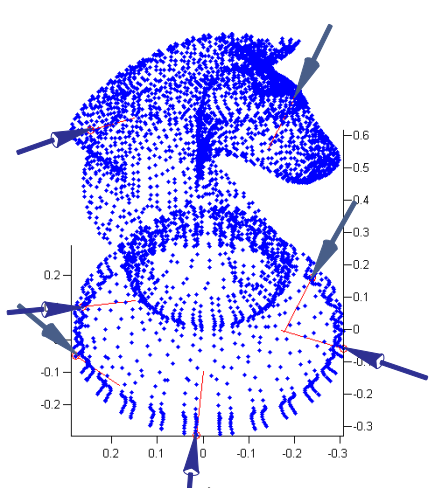

a)

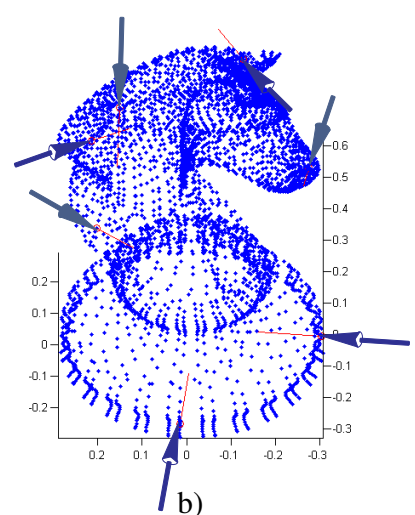

b)

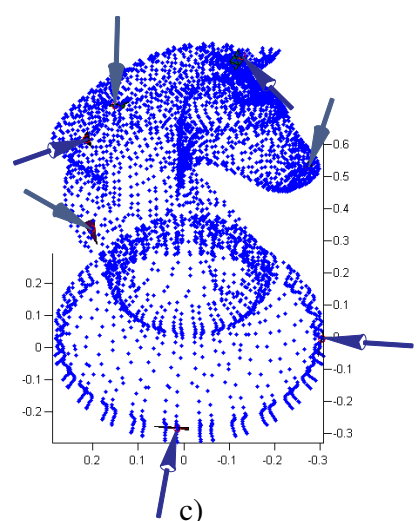

c)

Fig. 10. Example on a knight: a) Initial FC grasp, $Q=0.0003$ (Algorithm 1), b) Locally optimum FC grasp, $Q=0.077$ (Algorithm 2), c) Independent contact regions for each finger, $Q_{r}=0.058$ (Algorithm 3).

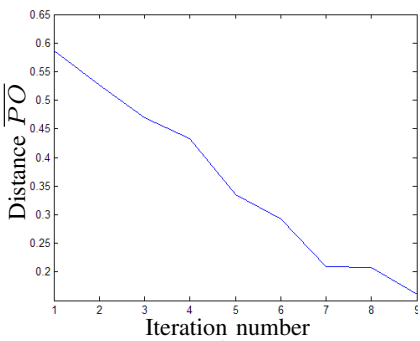

a)

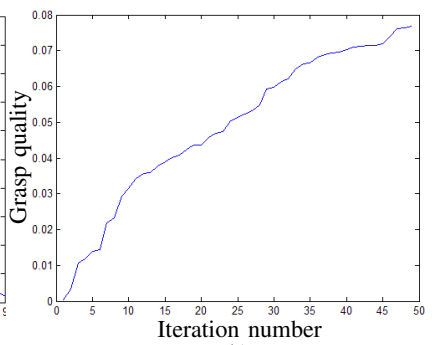

b)
Fig. 11. Performance in the search of a locally optimum FC grasp for the knight: a) Variation in the distance $\overline{P O}$, b) Increase in the grasp quality.

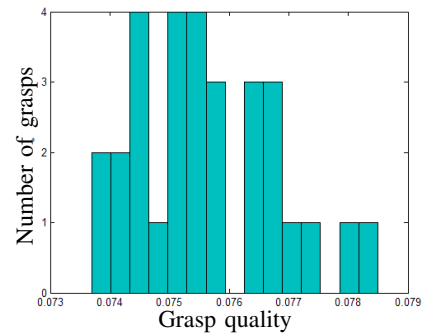

Fig. 12. Histogram with the grasp quality distribution for all the possible grasps within the independent contact region on the knight for $Q_{r}=0.058$ $(\alpha=0.75)$.

extension of the approach to consider frictional contacts is more complex, as the frictional model is nonlinear. In this case, the linearization of the friction cone requires suitable modifications in the presented algorithms, which will be addressed in future works.

\section{REFERENCES}

[1] A. Bicchi, "On the closure properties of robotic grasping," Int. J. Robotics Research, vol. 14, no. 4, pp. 319-344, 1995.

[2] Y. Liu, "Computing n-finger form-closure grasps on polygonal objects," Int. J. Robotics Research, vol. 19, no. 2, pp. 149-158, 2000.

[3] J. Cornellà and R. Suárez, "On computing form-closure grasps/fixtures for non-polygonal objects," in Proc. IEEE Int. Symp. Assembly and Task Planning, ISATP 2005, 2005, pp. 138-143.

[4] N. Niparnan and A. Sudsang, "Computing all force-closure grasps of 2D objects from contact point set," in Proc. IEEE/RSJ IROS 2006, 2006, pp. 1599-1604.

[5] J. Ponce, S. Sullivan, A. Sudsang, J. Boissonat, and J. Merlet, "On computing four-finger equilibrium and force-closure grasps of polyhedral objects," Int. J. Robotics Research, vol. 16, no. 1, pp. 1135, 1997.

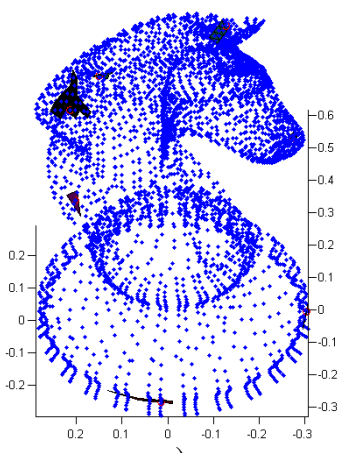

a)

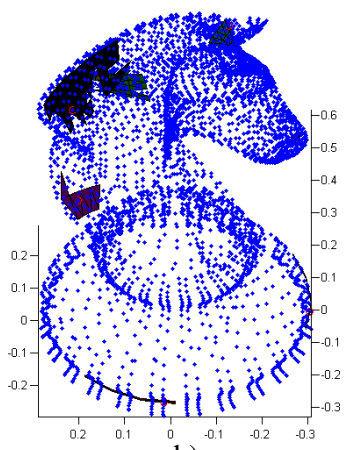

b)
Fig. 13. Independent contact regions on the knight with different minimum quality: a) $Q_{r}=0.039(\alpha=0.5)$, b) $Q_{r} \approx 0\left(\alpha=10^{-5}\right)$.

[6] D. Ding, Y. Liu, and S. Wang, "Computation of 3-D form-closure grasps," IEEE Trans. Robotics and Automation, vol. 17, no. 4, pp. 515-522, 2001.

[7] X. Zhu and J. Wang, "Synthesis of force-closure grasps on 3-D objects based on the Q distance," IEEE Trans. Robotics and Automation, vol. 19, no. 4, pp. 669-679, 2003.

[8] Y. Liu, M. Lam, and D. Ding, "A complete and efficient algorithm for searching 3-D form closure grasps in the discrete domain," IEEE Trans. Robotics, vol. 20, no. 5, pp. 805-816, 2004.

[9] V. Nguyen, "Constructing force-closure grasps," Int. J. Robotics Research, vol. 7, no. 3, pp. 3-16, 1988.

[10] J. Ponce and B. Faverjon, "On computing three-finger force-closure grasps of polygonal objects," IEEE Trans. Robotics and Automation, vol. 11 , no. 6, pp. 868-881, 1995.

[11] N. Pollard, "Closure and quality equivalence for efficient synthesis of grasps from examples," Int. J. Robotics Research, vol. 23, no. 6, pp. 595-614, 2004.

[12] J. Cornellà and R. Suárez, "Determining independent grasp regions on 2D discrete objects," in Proc. IEEE/RSJ IROS 2005, 2005, pp. 2936-2941.

[13] C. Ferrari and J. Canny, "Planning optimal grasps," in Proc. IEEE ICRA 1992, 1992, pp. 2290-2295.

[14] X. Zhu, H. Ding, and H. Li, "A quantitative measure for multifingered grasps," in Proc. IEEE/ASME Int. Conf. Advanced Intelligent Mechatronics 2001, 2001, pp. 213-219.

[15] B. Mishra, J. Schwartz, and M. Sharir, "On the existence and synthesis of multifinger positive grips," Algorithmica, vol. 2, no. 4, pp. 541-558, 1987.

[16] R. Murray, Z. Li, and S. Sastry, A Mathematical Introduction to Robotic Manipulation. Boca Ratón, Florida: CRC Press, 1994.

[17] Y. Liu, "Qualitative test and force optimization of 3-D frictional formclosure grasps using linear programming," IEEE Trans. Robotics and Automation, vol. 15, no. 1, pp. 163-173, 1999. 